\title{
Criteria for a Ring to have a Left Noetherian Largest Left Quotient Ring
}

\author{
V. V. Bavula ${ }^{1}$
}

Received: 29 September 2016 / Accepted: 22 June 2017 / Published online: 5 July 2017

(C) The Author(s) 2017. This article is an open access publication

\begin{abstract}
Criteria are given for a ring to have a left Noetherian largest left quotient ring. It is proved that each such a ring has only finitely many maximal left denominator sets. An explicit description of them is given. In particular, every left Noetherian ring has only finitely many maximal left denominator sets.
\end{abstract}

Keywords Goldie's Theorem · The left quotient ring of a ring · The largest left quotient ring of a ring - A maximal left denominator set - The left localization radical of a ring . An Ore set · A left denominator set · The prime radical

Mathematics Subject Classification (2010) $16 \mathrm{P} 50 \cdot 16 \mathrm{P} 60 \cdot 16 \mathrm{P} 20 \cdot 16 \mathrm{U} 20$

\section{Introduction}

In this paper, module means a left module, and the following notation is fixed:

- $\quad R$ is a ring with 1 and $R^{*}$ is its group of units;

- $\quad \mathcal{C}=\mathcal{C}_{R}$ is the set of regular elements of the ring $R$ (i.e. $\mathcal{C}$ is the set of non-zero-divisors of the ring $R$ );

- $Q=Q(R):=Q_{l, c l}(R):=\mathcal{C}^{-1} R$ is the left quotient ring (the classical left ring of fractions) of the ring $R$ (if it exists) and $Q^{*}$ is the group of units of $Q$;

- $\quad \mathfrak{n}=\mathfrak{n}_{R}$ is the prime radical of $R, v \in \mathbb{N} \cup\{\infty\}$ is its nilpotency degree $\left(\mathfrak{n}^{v} \neq 0\right.$ but $\left.\mathfrak{n}^{\nu+1}=0\right)$ and $\mathcal{N}_{i}:=\mathfrak{n}^{i} / \mathfrak{n}^{i+1}$ for $i \in \mathbb{N}$;

- $\bar{R}:=R / \mathfrak{n}$ and $\pi: R \rightarrow \bar{R}, r \mapsto \bar{r}=r+\mathfrak{n}$;

Presented by Kenneth Goodearl.

\section{V. Bavula}

v.bavula@sheffield.ac.uk

1 Department of Pure Mathematics, University of Sheffield, Hicks Building, Sheffield S3 7RH, UK 
- $\quad \overline{\mathcal{C}}:=\mathcal{C}_{\bar{R}}$ is the set of regular elements of the ring $\bar{R}$ and $\bar{Q}:=\overline{\mathcal{C}}^{-1} \bar{R}$ is its left quotient ring;

- $\quad \tilde{\mathcal{C}}:=\pi(\mathcal{C}), \widetilde{Q}:=\widetilde{\mathcal{C}}^{-1} \bar{R}$ and $\mathcal{C}^{\dagger}:=\mathcal{C}_{\widetilde{Q}}$ is the set of regular elements of the ring $\widetilde{Q}$;

- $\quad S_{l}=S_{l}(R)$ is the largest left Ore set of $R$ that consists of regular elements and $Q_{l}=$ $Q_{l}(R):=S_{l}(R)^{-1} R$ is the largest left quotient ring of $R$ [4, Theorem 2.1];

- $\quad \widetilde{S}_{l}:=\pi\left(S_{l}(R)\right)=\left\{s+\mathfrak{n} \mid s \in S_{l}(R)\right\}, \widetilde{Q}_{l}:=\widetilde{S}_{l}^{-1} \frac{\pi}{R}$ and $\mathcal{C}^{\dagger}:=\mathcal{C}_{\widetilde{Q}_{l}}$ (if the left quotient ring $Q$ of $R$ exists then $Q=Q_{l}$ and so $\mathcal{C}^{\dagger}=\mathcal{C}_{\widetilde{Q}_{l}}=\mathcal{C}_{\widetilde{Q}}$ );

- $\operatorname{Ore}_{l}(R):=\{S \mid S$ is a left Ore set in $R\}$;

- $\operatorname{Den}_{l}(R):=\{S \mid S$ is a left denominator set in $R\}$;

- $\operatorname{Ass}_{l}(R):=\left\{\operatorname{ass}(S) \mid S \in \operatorname{Den}_{l}(R)\right\}$ where $\operatorname{ass}(S):=\{r \in R \mid s r=0$ for some $s=$ $s(r) \in S\}$ (an ideal of $R)$;

- $\operatorname{Den}_{l}(R, \mathfrak{a}):=\left\{S \in \operatorname{Den}_{l}(R) \mid \operatorname{ass}(S)=\mathfrak{a}\right\}$ where $\mathfrak{a} \in \operatorname{Ass}_{l}(R)$

- For each $\mathfrak{a} \in \operatorname{Ass}_{l}(R)$, let $S_{\mathfrak{a}}=S_{\mathfrak{a}}(R)=S_{l, \mathfrak{a}}(R)$ is the largest element of the poset $\left(\operatorname{Den}_{l}(R, \mathfrak{a}), \subseteq\right)$ and $Q_{\mathfrak{a}}(R):=Q_{l, \mathfrak{a}}(R):=S_{\mathfrak{a}}^{-1} R$ is the largest left quotient ring associated to $\mathfrak{a}, S_{\mathfrak{a}}$ exists, [4];

Criteria for a Ring to have a Left Noetherian Largest Left Quotient Ring Stafford [12] obtained a criterion to determine when a Noetherian ring is its own quotient ring. Chatters and Hajarnavis [8] obtained necessary and sufficient conditions for a Noetherian ring which is a finite module over its centre to have a quotient ring. In [6], criteria are given for a ring $R$ to have a left Noetherian classical left quotient ring $Q(R)$. The aim of the paper is to give two criteria for a ring $R$ to have a left Noetherian largest left quotient ring (Theorem 1.1 and Theorem 1.2).

Theorem 1.1 Let $R$ be a ring. The following statements are equivalent.

1. The largest left quotient ring $Q_{l}(R)$ of $R$ is a left Noetherian ring.

2. (a) $\widetilde{S}_{l} \subseteq \overline{\mathcal{C}}$.

(b) $\widetilde{Q}_{l}=\widetilde{S}_{l}^{-1} \bar{R}$ is a left Noetherian ring.

(c) The prime radical $\mathfrak{n}$ of $R$ is a nilpotent ideal of the ring $R$.

(d) The $\widetilde{Q}_{l}$-modules $\widetilde{S}_{l}^{-1} \mathcal{N}_{i}, i=1, \ldots, v$, are finitely generated (where $v$ is the nilpotency degree of $\mathfrak{n}$ and $\left.\mathcal{N}_{i}:=\mathfrak{n}^{i} / \mathfrak{n}^{i+1}\right)$.

Remark The condition (a) above implies that the set $\widetilde{S}_{l}$ is a left denominator set of the ring $\bar{R}$, and so the ring $\widetilde{Q}_{l}$ exists.

Let $\mathfrak{n}$ and $\mathfrak{n}_{Q_{l}}$ be the prime radicals of the rings $R$ and $Q_{l}$, respectively. Their powers $\left\{\mathfrak{n}^{i}\right\}_{i \geq 0}$ and $\left\{\mathfrak{n}_{Q_{l}}\right\}_{i \geq 0}$ determine the (descending) prime radical filtrations on $R$ and $Q_{l}$, respectively. Let gr $R:=\bar{R} \oplus \mathfrak{n} / \mathfrak{n}^{2} \oplus \cdots$ and gr $Q_{l}:=Q_{l} / \mathfrak{n}_{Q_{l}} \oplus \mathfrak{n}_{Q_{l}} / \mathfrak{n}_{Q_{l}}^{2} \oplus \cdots$ be the associated graded rings. The second criterion is given in terms of the ring gr $R$.

Theorem 1.2 Let $R$ be a ring. The following statements are equivalent.

1. The ring $R$ has a left Noetherian largest left quotient ring ${\underset{\sim}{\mathcal{S}}}_{l}$.

2. The set $\widetilde{S}_{l}$ is a left denominator set of the ring gr $R, \widetilde{S}_{l} \subseteq \overline{\mathcal{C}}, \widetilde{S}_{l}^{-1}$ gr $R$ is a left Noetherian ring and $\mathfrak{n}$ is a nilpotent ideal.

If one of the equivalent conditions holds then $\operatorname{gr} Q_{l} \simeq \widetilde{S}_{l}^{-1} \operatorname{gr} R$ where $\operatorname{gr} Q_{l}:=\widetilde{Q} \oplus$ $\mathfrak{n}_{Q_{l}} / \mathfrak{n}_{Q_{l}}^{2} \oplus \cdots$ is the associated graded ring with respect to the prime radical filtration $\left\{\mathfrak{n}_{Q_{l}}^{i}\right\}_{i \geq 0}$. In particular, the ring gr $Q_{l}$ is a left Noetherian ring. 
Finiteness of the Set $\max . \operatorname{Den}_{l}(R)$ for a Ring $R$ with a Left Noetherian Largest

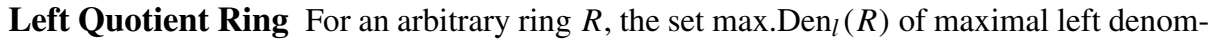

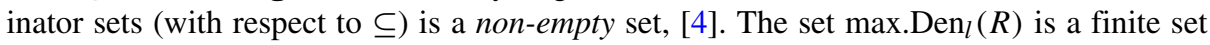
if the left quotient ring $Q(R)$ of $R$ is a left Noetherian, [6], the cases where $Q(R)$ is a semisimple ring or a left Artinian ring were considered in [2] and [5], respectively.

Theorem 1.3 Let $R$ be a ring such that its largest left quotient ring $Q_{l}(R)$ is a left Noetherian ring. Then $\left|\max _{\text {Den }}(R)\right|<\infty$. Moreover, $\left|\max _{l} \operatorname{Den}_{l}(R)\right| \leq s=\mid \max$ Den $_{l}(\bar{R}) \mid$ where $\bar{Q} \simeq \prod_{i=1}^{s} \bar{Q}_{i}$ and $\bar{Q}_{i}$ are simple Artinian rings (see Theorem 2.3.(3)).

A proof of Theorem 1.3 is given in Section 4. The next corollary follows at once from Theorem 1.3.

Corollary 1.4 Every left Noetherian ring has only finitely many maximal left denominator sets.

Proof Let $R$ be a left Noetherian ring. Then so is $Q_{l}$ and the result follows from Theorem 1.3.

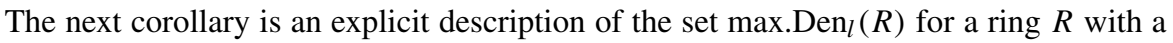
left Noetherian largest left quotient ring $Q_{l}(R)$.

Corollary 1.5 Let $R$ be a ring such that its largest left quotient ring $Q_{l}(R)$ is a left Noetherian ring. For each $i=1, \ldots, s$, let $p_{i}: R \rightarrow \bar{Q}_{i}$ be the natural projection (see Eq. 8 and Theorem 1.3), $\bar{Q}_{i}^{*}$ be the group of units of the simple Artinian ring $\bar{Q}_{i}$, $S_{i}^{\prime}$ be the largest element (w.r.t. $\subseteq$ ) of the set $D_{i}=\left\{S^{\prime} \in \operatorname{Den}_{l}(R) \mid p_{i}\left(S^{\prime}\right) \subseteq \bar{Q}_{i}^{*}\right\}$. Then

1. $\max \operatorname{Den}_{l}(R)$ is the set of maximal elements (w.r.t. $\subseteq$ ) of the set $\left\{S_{1}^{\prime}, \ldots, S_{s}^{\prime}\right\}$.

2. For all $i=1, \ldots, s, S_{l} \subseteq S_{i}^{\prime}$.

3. The rings $S_{i}^{\prime-1} R$ are left Noetherian where $i=1, \ldots, s$. In particular, the rings $S^{-1} R$

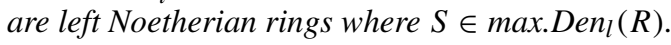

- Corollary 2.4 is a criterion for a ring to have a left Noetherian largest left quotient ring $Q_{l}$ such that the factor ring $Q_{l} / \mathfrak{n}_{Q_{l}}$ is a semisimple ring (or $\widetilde{Q}_{l}$ is a semisimple ring; or $\widetilde{S}_{l}=\overline{\mathcal{C}}$; or $S_{l}=\pi^{-1}(\overline{\mathcal{C}})$ ).

- Theorem 3.4 is a criterion for a ring $R$ to have a left Noetherian largest left quotient ring $Q_{l}$ such that the factor ring $Q_{l} / \mathfrak{n}_{Q_{l}}$ is a semisimple ring or a left Artinian ring.

- Theorem 4.2 is a criterion for a ring $R$ to have a left Noetherian ring such that $\left|\max _{\operatorname{Den}}(R)\right|=\left|\max _{l} \cdot \operatorname{Den}_{l}(\bar{R})\right|$ (recall that, in general, $\left|\max \cdot \operatorname{Den}_{l}(R)\right| \leq$ $\left|\max _{\operatorname{Lin}}(\bar{R})\right|$, Theorem 1.3).

The paper is organized as follows. In Section 2, many properties of a ring $R$ with a left Noetherian largest left quotient ring are proven (Theorem 2.3). In particular, the implication $(1 \Rightarrow 2)$ of Theorem 2.3 is proven. In Section 3, proofs of Theorem 1.1 and Theorem 3.4 are given.

\section{Rings with Left Noetherian Largest Left Quotient Ring}

At the beginning of this section, we collect necessary results that are used in the proofs of this paper. More results on localizations of rings (and some of the missed standard 
definitions) the reader can find in $[9,13]$ and [10]. In this section, we establish many properties of rings with left Noetherian largest left quotient ring (Theorem 2.3). In particular, the implication $(1 \Rightarrow 2)$ of Theorem 1.1 is proven (Theorem 2.3.(2)). A criterion is given (Corollary 2.4) for the ring $\widetilde{Q}_{l}$ to be a semisimple ring or a left Artinian ring.

\section{The Largest Regular Left Ore Set and the Largest Left Quotient Ring of a Ring}

Let $R$ be a ring. A multiplicatively closed subset $S$ of $R$ or a multiplicative subset of $R$ (i.e. a multiplicative sub-semigroup of $(R, \cdot)$ such that $1 \in S$ and $0 \notin S)$ is said to be a left Ore set if it satisfies the left Ore condition: for each $r \in R$ and $s \in S, S r \bigcap R s \neq \emptyset$. Let $\operatorname{Ore}_{l}(R)$ be the set of all left Ore sets of $R$. For $S \in \operatorname{Ore}_{l}(R)$, ass $(S):=\{r \in R \mid s r=0$ for some $s \in$ $S\}$ is an ideal of the ring $R$. A left Ore set $S$ is called a left denominator set of the ring $R$ if $r s=0$ for some elements $r \in R$ and $s \in S$ implies $t r=0$ for some element $t \in S$, i.e. $r \in \operatorname{ass}(S)$. Let $\operatorname{Den}_{l}(R)$ be the set of all left denominator sets of $R$. For $S \in \operatorname{Den}_{l}(R)$, let $S^{-1} R=\left\{s^{-1} r \mid s \in S, r \in R\right\}$ be the left localization of the ring $R$ at $S$ (the left quotient ring of $R$ at $S$ ). Let us stress that in Ore's method of localization one can localize precisely at left denominator sets.

In general, the set $\mathcal{C}$ of regular elements of a ring $R$ is neither left nor right Ore set of the ring $R$ and as a result neither left nor right classical quotient ring $\left(Q_{l, c l}(R):=\mathcal{C}^{-1} R\right.$ and $\left.Q_{r, c l}(R):=R \mathcal{C}^{-1}\right)$ exists. There exists the largest regular left Ore set $S_{0}=S_{l, 0}=S_{l}=S_{l, 0}(R)$, [4]. This means that the set $S_{l, 0}(R)$ is a left Ore set of the ring $R$ that consists of regular elements (i.e., $S_{l, 0}(R) \subseteq \mathcal{C}$ ) and contains all the left Ore sets in $R$ that consist of regular elements. Also, there exists the largest regular right (resp. left and right) Ore set $S_{r, 0}(R)$ (resp. $S_{l, r, 0}(R)$ ) of the ring $R$. In general, all the sets $\mathcal{C}, S_{l, 0}(R)$, $S_{r, 0}(R)$ and $S_{l, r, 0}(R)$ are distinct, for example, when $R=\mathbb{I}_{1}=K\left\langle x, \partial, \int\right\rangle$ is the ring of polynomial integro-differential operators over a field $K$ of characteristic zero, [1]. In [1], these four sets are found for $R=\mathbb{I}_{1}$.

Definition, [1, 4]. The ring

$$
Q_{l}(R):=S_{l, 0}(R)^{-1} R
$$

(respectively, $Q_{r}(R):=R S_{r, 0}(R)^{-1}$ and $Q_{l, r}(R):=S_{l, r, 0}(R)^{-1} R \simeq R S_{l, r, 0}(R)^{-1}$ ) is called the largest left (respectively, right and two-sided) quotient ring of the ring $R$.

In general, the rings $Q_{l}(R), Q_{r}(R)$ and $Q(R)_{l, r}$ are not isomorphic, for example, when $R=\mathbb{I}_{1}$, [1]. Furthermore, neither left nor right classical quotient ring exists for $\mathbb{I}_{1}$. Michler and Müller [11] mentioned that the ring $R$ contains a unique maximal (left and right) Ore set of regular elements $S_{l, r, 0}(R)$ and called the ring $Q_{l, r}(R)$ the total quotient ring of $R$.

The next theorem gives various properties of the ring $Q_{l}(R)$. In particular, it describes its group of units.

\section{Theorem 2.1 ([4])}

1. $S_{0}\left(Q_{l}(R)\right)=Q_{l}(R)^{*}$ and $S_{0}\left(Q_{l}(R)\right) \cap R=S_{0}(R)$.

2. $Q_{l}(R)^{*}=\left\langle S_{0}(R), S_{0}(R)^{-1}\right\rangle$, i.e. the group of units of the ring $Q_{l}(R)$ is generated by the sets $S_{0}(R)$ and $S_{0}(R)^{-1}:=\left\{s^{-1} \mid s \in S_{0}(R)\right\}$.

3. $Q_{l}(R)^{*}=\left\{s^{-1} t \mid s, t \in S_{0}(R)\right\}$.

4. $Q_{l}\left(Q_{l}(R)\right)=Q_{l}(R)$.

For a ring $R$, let $Q_{U}(R)$ be its maximal left ring of quotients (in the sense of Utumi). In [7], the class of rings $\mathcal{U}:=\left\{R \mid R \cap Q_{U}(R)^{*}=R^{*}\right\}$ is introduced where $Q_{U}(R)^{*}$ and $R^{*}$ are the groups of units of the rings $Q_{U}(R)$ and $R$, respectively. In view of Theorem 2.1.(1), 
it would be interesting to investigate connections of $Q_{l}(R)$ with the concept of the left ring hull of $R$ introduced in [7].

Properties of the Maximal Left Quotient Rings of a Ring The next theorem describes various properties of the maximal left quotient rings of a ring, in particular, their groups of units and their largest left quotient rings.

Theorem 2.2 ([4]) Let $S \in \max _{\text {Den }}(R), A=S^{-1} R, A^{*}$ be the group of units of the ring $A ; \mathfrak{a}:=\operatorname{ass}(S), \pi_{\mathfrak{a}}: R \rightarrow R / \mathfrak{a}, a \mapsto a+\mathfrak{a}$, and $\sigma_{\mathfrak{a}}: R \rightarrow A, r \mapsto \frac{r}{1}$. Then

1. $S=S_{\mathfrak{a}}(R), S=\pi_{\mathfrak{a}}^{-1}\left(S_{0}(R / \mathfrak{a})\right), \pi_{\mathfrak{a}}(S)=S_{0}(R / \mathfrak{a})$ and $A=S_{0}(R / \mathfrak{a})^{-1} R / \mathfrak{a}=$ $Q_{l}(R / \mathfrak{a})$.

2. $S_{0}(A)=A^{*}$ and $S_{0}(A) \cap(R / \mathfrak{a})=S_{0}(R / \mathfrak{a})$.

3. $S=\sigma_{a}^{-1}\left(A^{*}\right)$.

4. $A^{*}=\left\langle\pi_{\mathfrak{a}}(S), \pi_{\mathfrak{a}}(S)^{-1}\right\rangle$, i.e. the group of units of the ring $A$ is generated by the sets $\pi_{\mathfrak{a}}(S)$ and $\pi_{\mathfrak{a}}^{-1}(S):=\left\{\pi_{\mathfrak{a}}(s)^{-1} \mid s \in S\right\}$.

5. $A^{*}=\left\{\pi_{\mathfrak{a}}(s)^{-1} \pi_{\mathfrak{a}}(t) \mid s, t \in S\right\}$.

6. $Q_{l}(A)=A$ and $\operatorname{Ass}_{l}(A)=\{0\}$. In particular, if $T \in \operatorname{Den}_{l}(A, 0)$ then $T \subseteq A^{*}$.

Properties of a Ring with a Left Noetherian Largest Left Quotient Ring A ring $R$ is called a left Goldie ring if it satisfies ACC (the ascending chain condition) for left annihilators and contains no infinite direct sums of left ideals. Goldie's Theorem states that a ring $R$ has a semisimple left quotient ring iff the ring $R$ is a semiprime, left Goldie ring.

Theorem 2.3 Let $R$ be a ring such that its largest left quotient ring $Q_{l}$ is a left Noetherian ring. Let $\sigma: R \rightarrow Q_{l}, r \mapsto \frac{r}{1},\left(Q_{l} / \mathfrak{n}_{Q_{l}}\right)^{*}$ be the group of units of the ring $Q_{l} / \mathfrak{n}_{Q_{l}}$, and $\sigma^{\prime}: Q_{l} / \mathfrak{n}_{Q_{l}} \rightarrow Q\left(Q_{l} / \mathfrak{n}_{Q_{l}}\right), q+\mathfrak{n}_{Q_{l}} \mapsto \frac{q}{1}+\mathfrak{n}_{Q_{l}}$. Then

1. $\mathfrak{n}=R \cap \mathfrak{n}_{Q_{l}}, S_{l}^{-1} \mathfrak{n}=\mathfrak{n}_{Q_{l}},\left(S_{l}^{-1} \mathfrak{n}\right)^{i}=S_{l}^{-1} \mathfrak{n}^{i}$ for all $i \geq 1$, and $v=v_{Q_{l}}<\infty$ where $v$ and $v_{Q_{l}}$ are the nilpotency degrees of the prime radicals $\mathfrak{n}$ and $\mathfrak{n}_{Q_{l}}$, respectively.

2. (a) $S_{l}+\mathfrak{n} \subseteq S_{l}$.

(b) $\widetilde{S}_{l} \in \operatorname{Den}_{l}(\bar{R}, 0)$ where $\widetilde{S}_{l}:=\widetilde{S}_{l}(R):=\left\{s+\mathfrak{n} \mid s \in S_{l}(R)\right\}$. In particular, $\widetilde{S}_{l} \subseteq$ $S_{l}(\bar{R}) \subseteq \overline{\mathcal{C}}$.

(c) $\widetilde{Q}_{l}:=\widetilde{S}_{l}^{-1} \bar{R} \simeq Q_{l} / \mathfrak{n}_{Q_{l}}$ is a semiprime left Noetherian ring.

(d) $\mathfrak{n}$ is a nilpotent ideal of the ring $R$.

(e) The $\widetilde{Q}_{l}$-modules $\widetilde{S}_{l}^{-1}\left(\mathfrak{n}^{i} / \mathfrak{n}^{i+1}\right), i=1, \ldots, v$, are finitely generated (where $v$ is the nilpotency degree of $\mathfrak{n})$.

(f) For each elements $\bar{c} \in \widetilde{S}_{l}$, the left $\bar{R}$-module $\mathcal{N}_{i} / \mathcal{N}_{i} \bar{c}$ is $\widetilde{S}_{l}$-torsion where $\mathcal{N}_{i}:=$ $\mathfrak{n}^{i} / \mathfrak{n}^{i+1}$.

3. The ring $\bar{R}$ is a semiprime left Goldie ring and $\bar{Q}:=Q(\bar{R}) \simeq Q\left(Q_{l} / \mathfrak{n}_{Q_{l}}\right) \simeq Q\left(\widetilde{Q}_{l}\right)$ is a semisimple ring.

4. $1 \rightarrow 1+\mathfrak{n}_{Q_{l}} \rightarrow Q_{l}^{*} \stackrel{\pi_{Q_{l}}^{*}}{\rightarrow}\left(Q_{l} / \mathfrak{n}_{Q_{l}}\right)^{*} \rightarrow 1$ is a short exact sequence of group homomorphisms where $\pi_{Q_{l}}: Q_{l} \rightarrow Q_{l} / \mathfrak{n}_{Q_{l}}, q \mapsto q+\mathfrak{n}_{Q_{l}}$ and $\pi_{Q_{l}}^{*}:=\left.\pi_{Q_{l}}\right|_{Q_{l}^{*}}$.

5. $S_{l}=\sigma^{-1}\left(Q_{l}^{*}\right)=\left(\pi_{Q_{l}} \sigma\right)^{-1}\left(\left(Q_{l} / \mathfrak{n}_{Q_{l}}\right)^{*}\right)=\pi^{-1}\left(\widetilde{\sigma}^{-1}\left(\left(Q_{l} / \mathfrak{n}_{Q_{l}}\right)^{*}\right)\right.$ where $\widetilde{\sigma}: \bar{R} \rightarrow$ $Q_{l} / \mathfrak{n}_{Q_{l}}, \bar{r} \mapsto \frac{r}{1}+\mathfrak{n}_{Q_{l}}$, see Eq. 2.

6. Let $\mathcal{C}^{\dagger}:=\mathcal{C}_{\widetilde{Q}_{l}}$, i.e. $\mathcal{C}^{\dagger}=\mathcal{C}_{Q_{l} / \mathfrak{n}_{Q_{l}}}$ when we identify the rings $\widetilde{Q}_{l}$ and $Q_{l} / \mathfrak{n}_{Q_{l}}$ via the isomorphism in statement $2(c)$. Then $\mathcal{C}^{\dagger}=\sigma^{\prime-1}\left(Q\left(Q_{l} / \mathfrak{n}_{Q_{l}}\right)^{*}\right)$ and $\overline{\mathcal{C}}=\widetilde{\sigma}^{-1}\left(\mathcal{C}^{\dagger}\right)=$ $\left(\sigma^{\prime} \widetilde{\sigma}\right)^{-1}\left(Q\left(Q_{l} / \mathfrak{n}_{Q_{l}}\right)^{*}\right)$. 
Proof 1 . The prime radical $\mathfrak{n}_{Q_{l}}$ is a nilpotent ideal since the ring $Q_{l}$ is a left Noetherian ring. Then the intersection $R \cap \mathfrak{n}_{Q_{l}}$ is a nilpotent ideal of the ring $R$, hence $R \cap \mathfrak{n}_{Q_{l}} \subseteq \mathfrak{n}$. To establish the equality $R \cap \mathfrak{n}_{Q_{l}}=\mathfrak{n}$ it suffices to show that the factor $\operatorname{ring} R / R \cap \mathfrak{n}_{Q_{l}}$ has no nonzero nilpotent ideals. Suppose that $\bar{I}$ is a nilpotent ideal of the ring $R / R \cap \mathfrak{n}_{Q_{l}}$ then its preimage $I$ under the epimorphism $R \rightarrow R / R \cap \mathfrak{n}_{Q_{l}}, r \mapsto r+R \cap \mathfrak{n}_{Q_{l}}$, is a nilpotent ideal of the ring $R$ since the ideal $R \cap \mathfrak{n}_{Q_{l}}$ is a nilpotent ideal. We have to show that $\bar{I}=0$. The left ideal $S_{l}^{-1} I$ of $Q_{l}$ is an ideal of the ring $Q_{l}$ since the ring $Q_{l}$ is a left Noetherian ring. Then $I S_{l}^{-1}:=\left\{i s^{-1} \mid i \in I, s \in S_{l}\right\} \subseteq S_{l}^{-1} I$ and so

$$
\left(S_{l}^{-1} I\right)^{i} \subseteq S_{l}^{-1} I^{i}, \quad i \geq 1 .
$$

So, $S_{l}^{-1} I$ is a nilpotent ideal of the ring $Q_{l}$. Hence, $S_{l}^{-1} I \subseteq \mathfrak{n}_{Q_{l}}$ and $I \subseteq R \cap S_{l}^{-1} I \subseteq$ $R \cap \mathfrak{n}_{Q_{l}}$. Therefore, $\bar{I}=0$, as required. Clearly,

$$
S_{l}^{-1} \mathfrak{n}=S_{l}^{-1}\left(R \cap \mathfrak{n}_{Q_{l}}\right)=S_{l}^{-1} R \cap S_{l}^{-1} \mathfrak{n}_{Q_{l}}=Q_{l} \cap \mathfrak{n}_{Q_{l}}=\mathfrak{n}_{Q_{l}},
$$

and $\left(S_{l}^{-1} \mathfrak{n}\right)^{i}=S_{l}^{-1} \mathfrak{n}^{i}$ for $i \geq 1$. In particular, $v=v_{\mathfrak{n}_{Q_{l}}}<\infty$.

2(a) Let $c \in S_{l}$ and $n \in \mathfrak{n}$. Then the element $c^{-1} n \in S_{l}^{-1} \mathfrak{n}=\mathfrak{n}_{Q_{l}}$ (statement 1) is a nilpotent element of the ring $Q_{l}$ and so the element $1+c^{-1} n$ is a unit of the ring $Q_{l}$. Now,

$$
c+n=c\left(1+c^{-1} n\right) \in Q_{l}^{*},
$$

and so $c+n \in R \cap Q_{l}^{*}=S_{l}$ (Theorem 2.1.(1)).

2(b,c) Since $\mathfrak{n}=R \cap \mathfrak{n}_{Q_{l}}$ (statement 1 ), there is a commutative diagram of ring homomorphisms

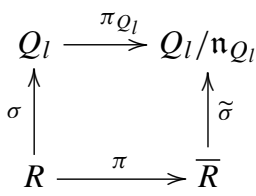

where the horizontal maps are natural epimorphisms and the vertical maps are natural monomorphisms (where $\widetilde{\sigma}(\bar{r}):=\frac{\bar{r}}{1}=\frac{r}{1}+\mathfrak{n}_{Q_{l}}$ ). Since

$$
Q_{l} / \mathfrak{n}_{Q_{l}}=\left\{\pi(c)^{-1} \bar{r} \mid c \in S_{l}, \bar{r} \in \bar{R}\right\},
$$

we see that $\widetilde{S}_{l}=\pi\left(S_{l}\right) \in \operatorname{Den}_{l}(\bar{R}, 0)$ and $Q_{l} / \mathfrak{n}_{Q_{l}} \simeq \widetilde{S}_{l}^{-1} \bar{R}$ is a semiprime left Noetherian ring.

2(d) The statement (d) follows from statement 1.

2(e) By the statement (c), $\widetilde{Q}_{l} \simeq Q_{l} / \mathfrak{n}_{Q_{l}}$. The ring $Q_{l}$ is a left Noetherian ring. Hence, the $Q_{l} / \mathfrak{n}_{Q_{l}}$-modules

$$
\mathfrak{n}_{Q_{l}}^{i} / \mathfrak{n}_{Q_{l}}^{i+1} \simeq\left(S_{l}^{-1} \mathfrak{n}\right)^{i} /\left(S_{l}^{-1} \mathfrak{n}\right)^{i+1} \simeq S_{l}^{-1} \mathfrak{n}^{i} / S_{l}^{-1} \mathfrak{n}^{i+1} \simeq \widetilde{S}_{l}^{-1}\left(\mathfrak{n}^{i} / \mathfrak{n}^{i+1}\right)
$$

are finitely generated where $i=1, \ldots, v$.

2(f) For each $i=1, \ldots, v$, the left $Q_{l}$-module/ $\widetilde{Q}_{l}$-module $\mathcal{N}_{i} / \mathcal{N}_{i} \bar{c}$ is $\widetilde{S}_{l}$-torsion since

$$
\begin{aligned}
\widetilde{S}_{l}^{-1}\left(\mathcal{N}_{i} / \mathcal{N}_{i} \bar{c}\right) & =\widetilde{S}_{l}^{-1}\left(\mathfrak{n}^{i} /\left(\mathfrak{n}^{i} c+\mathfrak{n}^{i+1}\right)\right)=S_{l}^{-1}\left(\mathfrak{n}^{i} /\left(\mathfrak{n}^{i} c+\mathfrak{n}^{i+1}\right)\right) \\
& =S_{l}^{-1} \mathfrak{n}^{i} /\left(S_{l}^{-1} \mathfrak{n}^{i} c+S_{l}^{-1} \mathfrak{n}^{i+1}\right)=S_{l}^{-1} \mathfrak{n}^{i} / S_{l}^{-1} \mathfrak{n}^{i}=0 .
\end{aligned}
$$

4. Statement 4 follows from the fact that $\mathfrak{n}_{Q_{l}}$ is a nilpotent ideal of the ring $Q_{l}$. 
3. By statement 2 (c), the ring $\widetilde{Q}_{l} \simeq Q_{l} / \mathfrak{n}_{Q_{l}}$ is a semiprime left Noetherian ring. In particular, it is a semiprime left Goldie ring and, by Goldie's Theorem, its left quotient ring $Q\left(\widetilde{Q}_{l}\right) \simeq Q\left(Q_{l} / \mathfrak{n}_{Q_{l}}\right)$ is a semisimple ring. Since $\bar{R} \subseteq Q_{l} / \mathfrak{n}_{Q_{l}} \simeq \widetilde{S}_{l}^{-1} \bar{R}$ (statement 2(c)) we have $Q(\bar{R})=Q\left(\widetilde{S}_{l}^{-1} \bar{R}\right)$ is a semisimple ring. By Goldie's Theorem, the ring $\bar{R}$ is a semiprime left Goldie ring. So, we can extend the commutative diagram (2) to the commutative diagram (which is used in the proof of statement 6 )

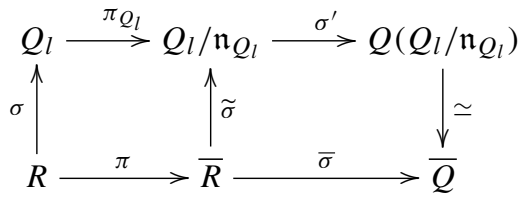

where the maps $\sigma^{\prime}$ and $\bar{\sigma}$ are monomorphisms, $\sigma^{\prime}\left(q+\mathfrak{n}_{Q_{l}}\right)=\frac{q+\mathfrak{n}_{Q_{l}}}{1}$ and $\bar{\sigma}(\bar{r})=\frac{\bar{r}}{1}$.

5. By Theorem 2.1.(1), $S_{l}=\sigma^{-1}\left(Q_{l}^{*}\right)$. By statement 4, $Q_{l}^{*}=\pi_{Q_{l}}^{-1}\left(\left(Q_{l} / \mathfrak{n}_{Q_{l}}\right)^{*}\right)$. Then, in view of the commutative diagram (2),

$$
\begin{aligned}
S_{l} & =\sigma^{-1} \pi_{Q_{l}}^{-1}\left(\left(Q_{l} / \mathfrak{n}_{Q_{l}}\right)^{*}\right)=\left(\pi_{Q_{l}} \sigma\right)^{-1}\left(\left(Q_{l} / \mathfrak{n}_{Q_{l}}\right)^{*}\right)=(\widetilde{\sigma} \pi)^{-1}\left(\left(Q_{l} / \mathfrak{n}_{Q_{l}}\right)^{*}\right) \\
& =\pi^{-1}\left(\widetilde{\sigma}^{-1}\left(\left(Q_{l} / \mathfrak{n}_{Q_{l}}\right)^{*}\right)\right) .
\end{aligned}
$$

6. By Theorem 2.1.(1), $\mathcal{C}^{\dagger}=\sigma^{\prime-1}\left(Q\left(Q_{l} / \mathfrak{n}_{Q_{l}}\right)^{*}\right)$ and $\overline{\mathcal{C}}=\bar{\sigma}^{-1}\left(\bar{Q}^{*}\right)=$ $\bar{\sigma}^{-1}\left(Q\left(Q_{l} / \mathfrak{n}_{Q_{l}}\right)^{*}\right)$ (statement 3$)$. Thus, the commutativity of the second square in the diagram (3) yields,

$$
\overline{\mathcal{C}}=\left(\sigma^{\prime} \widetilde{\sigma}\right)^{-1}\left(Q\left(Q_{l} / \mathfrak{n}_{Q_{l}}\right)^{*}\right)=\widetilde{\sigma}^{-1}\left(\mathcal{C}^{\dagger}\right)
$$

The next corollary is a criterion for a ring to have a left Noetherian largest left quotient ring $Q_{l}$ such that the factor ring $Q_{l} / \mathfrak{n}_{Q_{l}}$ is a semisimple ring (or $\widetilde{Q}_{l}$ is a semisimple ring; or $\widetilde{S}_{l}=\overline{\mathcal{C}}$; or $S_{l}=\pi^{-1}(\overline{\mathcal{C}})$ ).

Corollary 2.4 Let $R$ be a ring such that its largest left quotient ring $Q_{l}$ is a left Noetherian ring, we keep the notation of Theorem 2.3 and its proof. The following statements are equivalent (recall that $\widetilde{Q}_{l}=Q_{l} / \mathfrak{n}_{Q_{l}}$, Theorem 2.3.(2c)).

1. ${\widetilde{Q_{l}}}_{l}$ is a semisimple ring.

2. $\widetilde{Q}_{l}=Q\left(\widetilde{Q}_{l}\right)$.

3. $\overline{\mathcal{C}}=\widetilde{\sigma}^{-1}\left(\widetilde{Q}_{l}^{*}\right)$.

4. $S_{l}=\pi^{-1}(\overline{\mathcal{C}})$.

5. $\widetilde{S}_{l}=\overline{\mathcal{C}}$.

6. $\widetilde{Q}_{l}$ is a left Artinian ring.

Proof The implications $(1 \Rightarrow 2)$ and $(1 \Rightarrow 6)$ are obvious.

$(2 \Rightarrow 3)$ If $\widetilde{Q}_{l}=Q\left(\widetilde{Q}_{l}\right)$, i.e. the map $\sigma^{\prime}$ in Eq. 3 is an isomorphism, then the rings $\widetilde{Q}_{l} \simeq$ $Q_{l} / \mathfrak{n}_{Q_{l}}$ and $\bar{Q}$ are isomorphic, see the commutative diagram (3). Now, $\overline{\mathcal{C}}=\bar{\sigma}^{-1}\left(\bar{Q}^{*}\right)=$ $\widetilde{\sigma}^{-1}\left(\widetilde{Q}_{l}^{*}\right)$ where the first equality holds by Theorem 2.1.(1).

(3 $\Rightarrow 4$ ) By Theorem 2.3.(5). $S_{l}=\pi^{-1}\left(\widetilde{\sigma}^{-1}\left(\widetilde{Q}_{l}^{*}\right)\right)=\pi^{-1}(\overline{\mathcal{C}})$.

(4 $\Rightarrow$ 5) $\widetilde{S}_{l}=\pi\left(S_{l}\right)=\pi\left(\pi^{-1}(\overline{\mathcal{C}})\right)=\overline{\mathcal{C}}$ since the map $\pi$ is an epimorphism.

$(5 \Rightarrow 1)$ If $\widetilde{S}_{l}=\overline{\mathcal{C}}$ then by Eq. 3, $\widetilde{Q}_{l} \simeq \bar{Q}$ is a semisimple ring, by Theorem 2.3.(3).

$(6 \Rightarrow 1)$ This implication follows from Theorem 2.3.(2c). 


\section{Proof of Theorem 1.1 and Theorem 1.2}

In this section proofs of Theorem 1.1 and Theorem 1.2 are given.

Proof of Theorem 1.1. $(1 \Rightarrow 2)$ Theorem 2.3.(2).

$(1 \Leftarrow 2)$ (i) $S_{l}^{-1} \mathfrak{n}$ is an ideal of the ring $Q_{l}$ such that $Q_{l} / S_{l}^{-1} \mathfrak{n} \simeq \widetilde{Q}_{l}$ : Let $\sigma: R \rightarrow Q_{l}$, $r \mapsto \frac{r}{1}$. By the universal property of left localization, there is a ring homomorphism $\pi_{Q_{l}}$ : $Q_{l} \rightarrow \widetilde{Q}_{l}, c^{-1} r \mapsto \bar{c}^{-1} \bar{r}$, where $\bar{c}=c+\mathfrak{n}$ and $\bar{r}=r+\mathfrak{n}$, and we have the commutative diagram of ring homomorphisms

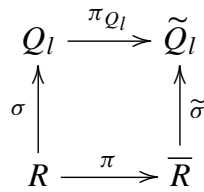

where $\tilde{\sigma}: \bar{r} \mapsto \frac{\bar{r}}{1}$ is a monomorphism and $\pi_{Q_{l}}$ is an epimorphism (by the very definition of $\pi_{Q_{l}}$ ). Applying the exact functor $S_{l}^{-1}(-)$ to the short exact sequence of $R$-modules $0 \rightarrow$ $\mathfrak{n} \rightarrow R \stackrel{\pi}{\rightarrow} \bar{R} \rightarrow 0$ we obtain the short exact sequence of $Q_{l}$-modules

$$
0 \rightarrow S_{l}^{-1} \mathfrak{n} \rightarrow Q_{l} \stackrel{\pi_{Q_{l}}}{\rightarrow} S_{l}^{-1} \bar{R}=\widetilde{S}_{l}^{-1} \bar{R}=\widetilde{Q}_{l} \rightarrow 0 .
$$

Therefore, $\operatorname{ker}\left(\pi_{Q_{l}}\right)=S_{l}^{-1} \mathfrak{n}$ is an ideal of $Q_{l}$ (since $\pi_{Q_{l}}$ is a ring homomorphism) such that $Q_{l} / S_{l}^{-1} \mathfrak{n} \simeq \widetilde{Q}_{l}$.

(ii) $\left(S_{l}^{-1} \mathfrak{n}\right)^{i}=S_{l}^{-1} \mathfrak{n}^{i}$ for $i \geq 1$ : This follows from (i).

(iii) The ring $Q_{l}$ is a left Noetherian ring: By localizing the descending chain of ideals of the ring $R$ :

$$
R \supseteq \mathfrak{n} \supseteq \mathfrak{n}^{2} \supseteq \cdots \supseteq \mathfrak{n}^{i} \supseteq \cdots \supseteq \mathfrak{n}^{v} \supseteq \mathfrak{n}^{v+1}=0
$$

we obtain a descending chain of ideals (by (ii)) of the ring $Q_{l}$ :

$$
Q_{l} \supseteq S_{l}^{-1} \mathfrak{n} \supseteq S_{l}^{-1} \mathfrak{n}^{2} \supseteq \cdots \supseteq S_{l}^{-1} \mathfrak{n}^{i} \supseteq \cdots \supseteq S_{l}^{-1} \mathfrak{n}^{\nu} \supseteq S_{l}^{-1} \mathfrak{n}^{\nu+1}=0 .
$$

By (i), $\widetilde{Q}_{l} \simeq Q_{l} / S_{l}^{-1} \mathfrak{n}$ is a left Noetherian $Q_{l}$-module since the ring $\widetilde{Q}_{l}$ is a left Noetherian ring, by the condition (b). For each $i=1, \ldots, v$, the left $Q$-module, $S_{l}^{-1} \mathfrak{n}^{i} / S_{l}^{-1} \mathfrak{n}^{i+1} \simeq$ $\left(S_{l}^{-1} \mathfrak{n}\right)^{i} /\left(S_{l}^{-1} \mathfrak{n}\right)^{i+1}$ is a $Q_{l} / S_{l}^{-1} \mathfrak{n}=\widetilde{Q}_{l}$-module, by (i). The $\widetilde{Q}_{l}$-modules

$$
S_{l}^{-1} \mathfrak{n}^{i} / S_{l}^{-1} \mathfrak{n}^{i+1} \simeq S_{l}^{-1}\left(\mathfrak{n}^{i} / \mathfrak{n}^{i+1}\right) \simeq \widetilde{S}_{l}^{-1}\left(\mathfrak{n}^{i} / \mathfrak{n}^{i+1}\right)=\widetilde{S}_{l}^{-1} \mathcal{N}_{i}
$$

are finitely generated, by the condition (d), hence Noetherian since the ring $\widetilde{Q}_{l}$ is a left Noetherian ring. Since all the factors of the finite filtration (5) are Noetherian $\widetilde{Q}_{l}$-modules/ $Q_{l}$-modules, the ring $Q_{l}$ is a left Noetherian ring.

\section{The Maximal Denominator Sets and the Maximal Left Localizations of a Ring} The set $\left(\operatorname{Den}_{l}(R), \subseteq\right)$ is a poset (partially ordered set). In [4], it is proved that the set $\max _{\operatorname{Den}}(R)$ of its maximal elements is a non-empty set.

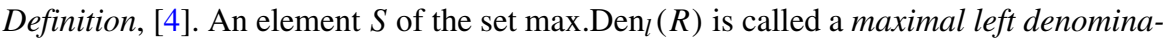
tor set of the ring $R$ and the ring $S^{-1} R$ is called a maximal left quotient ring of the ring $R$ or a maximal left localization ring of the ring $R$. The intersection

$$
\mathfrak{l}_{R}:=1 \cdot \operatorname{lrad}(R):=\bigcap_{S \in \max ^{2} \operatorname{Den}_{l}(R)} \operatorname{ass}(S)
$$

is called the left localization radical of the ring $R$, [4]. 
For a ring $R$, there is a canonical exact sequence

$$
0 \rightarrow \mathfrak{l}_{R} \rightarrow R \stackrel{\sigma}{\rightarrow} \prod_{S \in \max _{\operatorname{man}}(R)} S^{-1} R, \quad \sigma:=\prod_{S \in \max _{l} \cdot \operatorname{Den}_{l}(R)} \sigma_{S},
$$

where $\sigma_{S}: R \rightarrow S^{-1} R, r \mapsto \frac{r}{1}$.

\section{A Bijection Between the Sets $\max ^{-D_{e n}}(R)$ and $\max _{l} \operatorname{Den}_{l}\left(Q_{l}(R)\right)$}

Proposition 3.1 ([2]) Let $R$ be a ring, $S_{l}$ be the largest regular left Ore set of the ring $R$, $Q_{l}=S_{l}^{-1} R$ be the largest left quotient ring of the ring $R$, and $\mathcal{C}$ be the set of regular elements of the ring $R$. Then

1. $S_{l} \subseteq S$ for all $S \in \max \operatorname{Den}_{l}(R)$. In particular, $\mathcal{C} \subseteq S$ for all $S \in \max _{\operatorname{Den}}(R)$ provided $\mathcal{C}$ is a left Ore set.

2. Either max.Den $(R)=\{\mathcal{C}\}$ or, otherwise, $\mathcal{C} \notin \max _{l} \operatorname{Den}_{l}(R)$.

3. The map

$$
\max _{\operatorname{Den}}(R) \rightarrow \max _{\operatorname{Den}}\left(Q_{l}\right), \quad S \mapsto S Q_{l}^{*}=\left\{c^{-1} s \mid c \in S_{l}, s \in S\right\},
$$

is a bijection with the inverse $\mathcal{T} \mapsto \sigma^{-1}(\mathcal{T})$ where $\sigma: R \rightarrow Q_{l}, r \mapsto \frac{r}{1}$, and $S Q_{l}^{*}$ is the sub-semigroup of $\left(Q_{l}, \cdot\right)$ generated by the set $S$ and the group $Q_{l}^{*}$ of units of the ring $Q_{l}$, and $S^{-1} R=\left(S Q_{l}^{*}\right)^{-1} Q_{l}$.

4. If $\mathcal{C}$ is a left Ore set then the map

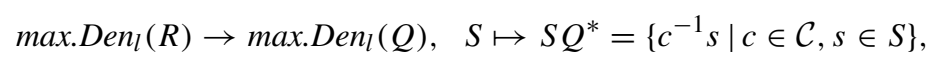

is a bijection with the inverse $\mathcal{T} \mapsto \sigma^{-1}(\mathcal{T})$ where $\sigma: R \rightarrow Q, r \mapsto \frac{r}{1}$, and $S Q^{*}$ is the sub-semigroup of $(Q, \cdot)$ generated by the set $S$ and the group $Q^{*}$ of units of the ring $Q$, and $S^{-1} R=\left(S Q^{*}\right)^{-1} Q$.

The Minimal Primes of the Rings $R, Q_{l}, \bar{Q}$ and $\widetilde{Q}_{l}$ For a $\operatorname{ring} R$ such that $Q_{l}$ is a left Noetherian ring, the next corollary shows that the localizations of $R$ at the maximal left denominator sets are left Noetherian rings and there are natural bijections between the sets of minimal primes of the rings $R, Q_{l}, \bar{Q}$ and $\widetilde{Q}_{l}$.

Corollary 3.2 Let $R$ be a ring such that $Q_{l}$ is a left Noetherian ring. Then

1. For every $S \in \max _{\text {.Den }}(R)$, the ring $S^{-1} R$ is a left Noetherian ring.

2. (a) The map $\operatorname{Min}(R) \rightarrow \operatorname{Min}(\bar{Q}), \mathfrak{p} \mapsto \overline{\mathcal{C}}^{-1}(\mathfrak{p} / \mathfrak{n})$, is a bijection with the inverse $\mathfrak{q} \mapsto(\bar{\sigma} \pi)^{-1}(\mathfrak{q})$ where $\bar{\sigma}: \bar{R} \rightarrow \bar{Q}, \bar{r} \mapsto \frac{\bar{r}}{1}$.

(b) The map $\operatorname{Min}(R) \rightarrow \min \left(\widetilde{Q}_{l}\right), \mathfrak{p} \mapsto \widetilde{S}_{l}^{-1}(\mathfrak{p} / \mathfrak{n})$, is a bijection with the inverse $\mathfrak{q} \mapsto \tau^{-1}(\mathfrak{q})$ where $\tau: R \rightarrow \widetilde{Q}_{l}, r \mapsto \frac{r+\mathfrak{p}}{1}$.

(c) The map $\operatorname{Min}(R) \rightarrow \operatorname{Min}\left(Q_{l}\right), \mathfrak{p} \mapsto S_{l}^{-1} \mathfrak{p}$, is a bijection with the inverse $\mathfrak{q} \mapsto$ $\mathfrak{q} \cap R$.

Proof 1. By Proposition 3.1.(1), $S_{l} \subseteq S$ for all $S \in \max \operatorname{Den}_{l}(R)$. The ring $Q_{l}:=S_{l}^{-1} R$ is a left Noetherian ring and the ring $S^{-1} R \simeq\left(S Q_{l}^{*}\right)^{-1} Q_{l}$ is a left localization of the ring

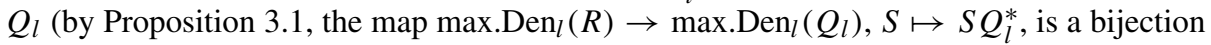
with $\left.S^{-1} R \simeq\left(S Q^{*}\right)^{-1} Q_{l}\right)$. Therefore, the ring $S^{-1} R$ is a left Noetherian ring since the ring $Q_{l}$ is so. 
2(a) The map $\operatorname{Min}(R) \rightarrow \operatorname{Min}(\bar{R}), \mathfrak{p} \mapsto \mathfrak{p} / \mathfrak{n}$, is a bijection with the inverse $\mathfrak{p}^{\prime} \mapsto$ $\pi^{-1}\left(\mathfrak{p}^{\prime}\right)$ where $\pi: R \rightarrow \bar{R}, r \mapsto r+\mathfrak{p}$. The ring $\bar{R}$ is a semiprime left Goldie ring such that $\bar{Q} \simeq Q\left(Q_{l} / \mathfrak{n}_{Q_{l}}\right)$ is a semisimple ring (Theorem 2.3.(3)). Hence, the map $\min (\bar{R}) \rightarrow$ $\operatorname{Min}(\bar{Q}), \mathfrak{p}^{\prime} \mapsto \overline{\mathcal{C}}^{-1} \mathfrak{p}^{\prime}$, is a bijection with the inverse $\mathfrak{q} \mapsto \bar{\sigma}^{-1}(\mathfrak{q})$ where $\bar{\sigma}: \bar{R} \rightarrow \bar{Q}$, $\bar{r} \mapsto \bar{r}$. Now, the statement (a) follows.

(b) The ring $\widetilde{Q}_{l}$ is a semiprime left Goldie ring (by Theorem 2.3.(3)) and $Q\left(\widetilde{Q}_{l}\right) \simeq \widetilde{Q}$ is a semisimple ring (Theorem 2.3.(3)). So, the map $\operatorname{Min}\left(\widetilde{Q}_{l}\right) \rightarrow \operatorname{Min}(\bar{Q}), P \mapsto \bar{Q} \otimes \widetilde{Q}_{l} P$, is a bijection with the inverse $P^{\prime} \mapsto P^{\prime} \cap \widetilde{Q}_{l}$. Now, the statement (b) follows from the statement (a).

(c) By Theorem 2.3.(2c), $\widetilde{Q}_{l} \simeq Q_{l} / \mathfrak{n}_{Q_{l}}$ and the map $\operatorname{Min}\left(Q_{l}\right) \rightarrow \operatorname{Min}\left(Q_{l} / \mathfrak{n}_{Q_{l}}\right), P \mapsto$ $P / \mathfrak{n}_{Q_{l}}$, is a bijection. Now, the statement (c) follows from the statement (b).

\section{Characterization of the Set $S_{l}(R)$ when $Q_{l}(R)$ is a Left Noetherian Ring}

Theorem 3.3 Let $R$ be a ring such that $Q_{l}(R)$ is a left Noetherian ring. Then

1. $S_{l}(R)=\sup \left\{S \mid S \in \operatorname{Den}_{l}(R, 0), \pi(S) \in \operatorname{Den}_{l}(\bar{R}, 0), S+\mathfrak{n} \subseteq S\right\}$.

2. $S_{l}(R)=\sup \left\{\pi^{-1}(T) \mid T \in \operatorname{Den}_{l}(\bar{R}, 0), \pi^{-1}(T) \in \operatorname{Den}_{l}(R, 0)\right\}$.

Proof 1 . Let $\mathbb{S}$ be the set $\{S\}$ on the RHS of the equality. Then $S \subseteq S_{l}$ for all $S \in \mathbb{S}$ (since $S \in \operatorname{Den}_{l}(R, 0)$ ) and $S_{l} \in \mathbb{S}$ since $\widetilde{S}_{l}=\pi\left(S_{l}\right) \in \operatorname{Den}_{l}(\bar{R}, 0)$ (Theorem 1.1(2a,b)) and $S_{l}+\mathfrak{n} \subseteq S_{l}$ (Theorem 2.3.(2a)).

2. Statement 2 follows from statement 1.

The next theorem is a criterion for a ring $R$ to have a left Noetherian largest left quotient ring $Q_{l}$ such that the factor ring $Q_{l} / \mathfrak{n}_{Q_{l}}$ is a semisimple ring.

Theorem 3.4 Let $R$ be a ring. The following statements are equivalent.

1. The ring $R$ has a left Noetherian largest left quotient ring $Q_{l}$ such that the factor ring $Q_{l} / \mathfrak{n}_{Q_{l}}$ is a semisimple ring.

2. The conditions of Theorem 1.1.(2) and one of the equivalent conditions of Corollary 2.4 hold.

3. The ring $R$ has a left Noetherian largest left quotient ring $Q_{l}$ such that the factor ring $Q_{l} / \mathfrak{n}_{Q_{l}}$ is a left Artinian ring.

Proof $(1 \Leftrightarrow 2)$ Theorem 1.1 and Corollary 2.4.

$(1 \Rightarrow 3)$ Trivial.

$(1 \Leftarrow 3)$ This implication follows from Theorem 2.3.(3).

Lemma 3.5 ([2]) Let $G$ be a monoid and $e \in G$ be its neutral element, $A=\bigoplus_{g \in G} A_{g}$ be a $G$-graded ring, $1 \in A_{e}, S \in \operatorname{Den}_{l}(A)$ and $\mathfrak{a}=\operatorname{ass}(S)$. If $S \subseteq A_{e}$ then the ring $S^{-1} A=$ $\bigoplus_{g \in G}\left(S^{-1} A\right)_{g}$ is a G-graded ring where $\left(S^{-1} A\right)_{g}=S^{-1} A_{g}:=\left\{s^{-1} a_{g} \mid s \in S, a_{g} \in A_{g}\right\}$, $S \in \operatorname{Den}_{l}\left(A_{e}\right)$ and $\mathfrak{a}=\bigoplus_{g \in G} \mathfrak{a}_{g}$ is a G-graded ideal of the ring A, i.e. $\mathfrak{a}_{g}=\mathfrak{a} \cap A_{g}$ for all $g \in G$. 
Suppose that a ring $R$ has a left Noetherian largest left quotient ring $Q_{l}$. By Theorem 2.3, the associated graded ring gr $Q_{l}:=Q_{l} / \mathfrak{n}_{Q_{l}} \oplus \mathfrak{n}_{Q_{l}} / \mathfrak{n}_{Q_{l}}^{2} \oplus \cdots$ is equal to

$$
\operatorname{gr} Q_{l}=\widetilde{Q}_{l} \oplus \widetilde{S}_{l}^{-1}\left(\mathfrak{n} / \mathfrak{n}^{2}\right) \oplus \cdots \oplus \widetilde{S}_{l}^{-1}\left(\mathfrak{n}^{\nu} / \mathfrak{n}^{\nu+1}\right)
$$

Proof of Theorem 1.2. $(1 \Rightarrow 2)$ Suppose that the ring $Q_{l}$ is a left Noetherian ring, and so the conditions of Theorem 2.3 and Theorem 1.1 hold. In particular, $\widetilde{S}_{l} \subseteq \overline{\mathcal{C}}$ and $\mathfrak{n}$ is a nilpotent ideal.

(i) $\widetilde{S}_{l} \in \operatorname{Ore}_{l}(\mathrm{gr} R)$ : It suffices to show that for given elements $\bar{c}=c+\mathfrak{n} \in \widetilde{S}_{l}$ and $r+\mathfrak{n}^{i+1} \in \mathfrak{n}^{i} / \mathfrak{n}^{i+1}$ where $c \in S_{l}, r \in \mathfrak{n}^{i}$ and $i=0,1, \ldots, v$, there are elements $\bar{c}^{\prime}=c^{\prime}+\mathfrak{n} \in$ $\widetilde{S}_{l}$ and $r^{\prime}+\mathfrak{n}^{i+1} \in \mathfrak{n}^{i} / \mathfrak{n}^{i+1}$ where $c^{\prime} \in S_{l}, r^{\prime} \in \mathfrak{n}^{i}$ such that $\bar{c}^{\prime}\left(r+\mathfrak{n}^{i+1}\right)=\left(r^{\prime}+\mathfrak{n}^{i+1}\right) \bar{c}$.

The case $i=0$ is obvious, by Theorem 1.1.(2b). So, we can assume that $i \geq 1$. By Theorem 2.3.(1),

$$
\mathfrak{n}^{i} S_{l}^{-1}:=\left\{n c^{-1} \mid n \in \mathfrak{n}^{i}, c \in S_{l}\right\} \subseteq S_{l}^{-1} \mathfrak{n}^{i} .
$$

So, $r c^{-1}=c^{-1} r^{\prime}$ for some elements $c^{\prime} \in S_{l}$ and $r^{\prime} \in \mathfrak{n}^{i}$, hence $c^{\prime} r=r^{\prime} c$. This equality implies the required one.

(ii) $\widetilde{S}_{l} \in \operatorname{Den}_{l}(\mathrm{gr} R)$ : Theorem 2.3.(2b,f).

(iii) gr $Q_{l} \simeq \widetilde{S}_{l}^{-1}$ gr $R$ : By Theorem 2.3.(1), $\mathfrak{n}_{Q_{l}}=S_{l}^{-1} \mathfrak{n}$. By Theorem 2.3.(2c), $Q_{l} / \mathfrak{n}_{Q_{l}} \simeq \widetilde{Q}_{l}$. Now, using Theorem 2.3.(1,2), we have

$$
\begin{aligned}
\operatorname{gr} Q_{l} & =\widetilde{Q}_{l} \oplus \cdots \oplus \mathfrak{n}_{Q_{l}}^{i} / \mathfrak{n}_{Q_{l}}^{i+1} \oplus \cdots=\widetilde{Q}_{l} \oplus \cdots \oplus S_{l}^{-1} \mathfrak{n}^{i} / S_{l}^{-1} \mathfrak{n}^{i+1} \oplus \cdots \\
& =\widetilde{Q}_{l} \oplus \cdots \oplus S_{l}^{-1}\left(\mathfrak{n}^{i} / \mathfrak{n}^{i+1}\right) \oplus \cdots=\widetilde{Q}_{l} \oplus \cdots \oplus \widetilde{S}_{l}^{-1}\left(\mathfrak{n}^{i} / \mathfrak{n}^{i+1}\right) \oplus \cdots \\
& \simeq \widetilde{S}_{l}^{-1} \operatorname{gr} R .
\end{aligned}
$$

(iv) $\widetilde{S}_{l}^{-1} \operatorname{gr} R$ is a left Noetherian ring: The ring $\widetilde{Q}_{l}$ is a left Noetherian ring (Theorem 2.3.(2c)) and the left $\widetilde{Q}_{l}$-modules $\widetilde{S}_{l}^{-1}\left(\mathfrak{n}^{i} / \mathfrak{n}^{i+1}\right) \simeq\left(S_{l}^{-1} \mathfrak{n}\right)^{i} /\left(S_{l}^{-1} \mathfrak{n}\right)^{i+1}$ are finitely generated where $i=1, \ldots, v$ (since $Q_{l}$ is a left Noetherian ring). Therefore, the left $\widetilde{Q}_{l}$-module $\operatorname{gr} Q_{l}=\widetilde{Q}_{l} \oplus \cdots \oplus \widetilde{S}_{l}^{-1}\left(\mathfrak{n}^{i} / \mathfrak{n}^{i+1}\right) \oplus \cdots \oplus \widetilde{S}_{l}^{-1}\left(\mathfrak{n}^{\nu} / \mathfrak{n}^{\nu+1}\right)$ is finitely generated, hence Noetherian. Since $\widetilde{Q}_{l} \subseteq$ gr $Q_{l}$, the ring gr $Q_{l}$ is a left Noetherian ring.

( $1 \Leftarrow 2$ ) It suffices to show that the conditions (a)-(d) of Theorem 1.1.(2) hold. The conditions (a) and (c) are given. The set $\widetilde{S}_{l}$ is a left denominator set of the $\mathbb{N}$-graded ring gr $R$ such that $\widetilde{S}_{l} \subseteq \bar{R}$. By Lemma 3.5, the ring $\widetilde{S}_{l}^{-1}$ gr $R=\widetilde{Q}_{l} \oplus \cdots \oplus \widetilde{S}_{l}^{-1} \mathcal{N}_{i} \oplus \cdots$ is an $\mathbb{N}$-graded ring and $\widetilde{S}_{l} \in \operatorname{Den}_{l}(\bar{R}, 0)$. The ring $\widetilde{Q}_{l}$ is a factor ring of the left Noetherian ring $\widetilde{S}_{l}^{-1}$ gr $R$, hence $\widetilde{Q}_{l}$ is a left Noetherian ring, i.e. the condition (c) holds.

The ring $\widetilde{S}_{l}^{-1}$ gr $R$ is left Noetherian, hence the $\widetilde{Q}_{l}$-modules $\widetilde{S}_{l}^{-1} \mathcal{N}_{i}$ are finitely generated, i.e. the condition (e) holds.

Corollary 3.6 Let $R$ be a ring with a left Noetherian largest left quotient ring $Q_{l}$ and $\tilde{\mathfrak{a}}:=\operatorname{ass}_{g r}\left(\widetilde{S}_{l}\right)$. Then the largest left quotient ring $Q_{l}(\mathrm{gr} R / \mathfrak{\mathfrak { a }})$ of the ring $\mathrm{gr} R / \tilde{\mathfrak{a}}$ is a left Noetherian ring.

Proof By Theorem 1.2.(2), the ring $\widetilde{S}_{l}^{-1}$ gr $R$ is a left Noetherian ring. The ring $Q_{l}(\operatorname{gr} R / \tilde{a})$ is a left localization of the ring $\widetilde{S}_{l}^{-1}$ gr $R$, hence is a left Noetherian ring. 


\section{Finiteness of the Set $\max \operatorname{Den}_{l}(R)$ when $Q_{l}(R)$ is a Left Noetherian Ring}

The aim of this section is to give a proof of Theorem 1.3.

Let $R$ be a ring. Let $S, T$ be submonoids of the multiplicative monoid $(R, \cdot)$. We denote by $S T$ the submonoid of $(R, \cdot)$ generated by $S$ and $T$. This notation should not be confused with the product of two sets which is not used in this paper. The next result is a criterion for the set $S T$ to be a left Ore (denominator) set, it is used at the final stage in the proof of Theorem 1.3.

\section{Lemma $4.1([3])$}

1. Let $S, T \in \operatorname{Ore}_{l}(R)$. If $0 \notin S T$ then $S T \in \operatorname{Ore}_{l}(R)$.

2. Let $S, T \in \operatorname{Den}_{l}(R)$. If $0 \notin S T$ then $S T \in \operatorname{Den}_{l}(R)$.

Proof of Theorem 1.3. Let $S \in \max ^{-D} \operatorname{Den}_{l}(R)$ and $\pi: R \rightarrow \bar{R}, r \mapsto \bar{r}:=r+\mathfrak{n}$.

(i) $\bar{S}:=\pi(S) \in \operatorname{Ore}_{l}(\bar{R})$ : This obvious since $S \cap \mathfrak{n}=\emptyset$.

(ii) $S_{l}(R) \subseteq S$ (Proposition 3.1.(1)).

(iii) $S^{-1} R$ is a left Noetherian ring: By (ii), $S^{-1} R$ is a left localization of the left Noetherian ring $Q_{l}$, hence $S^{-1} R$ is a left Noetherian ring.

(iv) $S^{-1} \mathfrak{n}$ is an ideal of $S^{-1} R$ such that $\left(S^{-1} \mathfrak{n}\right)^{i}=S^{-1} \mathfrak{n}^{i}$ for all $i \geq 1$ : By (iii), $S^{-1} \mathfrak{n}$ is an ideal of the ring $S^{-1} R$. In particular, $\mathfrak{n} S^{-1} \subseteq S^{-1} \mathfrak{n}$, hence $\left(S^{-1} \mathfrak{n}\right)^{i}=S^{-1} \mathfrak{n}$ for all $i \geq 1$.

(v) $\bar{S} \in \operatorname{Den}_{l}(\bar{R}):$ In view of (i), we have to show that if $\overline{r s}=0$ for some $\bar{r} \in \bar{R}$ and $\bar{s} \in \bar{S}$ then $\bar{t} \bar{r}=0$ for some element $\bar{t} \in \bar{S}$. The equality $\overline{r s}=0$ means that $n:=r s \in \mathfrak{n}$. Then $S^{-1} R \ni \frac{r}{1}=n s^{-1}=s_{1}^{-1} n_{1}$ for some $s_{1} \in S$ and $n_{1} \in \mathfrak{n}$, (by (iv)). Hence, $s_{2} s_{1} r=s_{2} n_{1} \in \mathfrak{n}$ for some $s_{2} \in S$, and so $\bar{t} \bar{r}=0$ where $\bar{t}=\bar{s}_{2} \bar{s}_{1} \in \bar{S}$.

The ring $\bar{R}$ is a semiprime left Goldie ring, $\bar{Q}=\prod_{i=1}^{s} \bar{Q}_{i}$ and $\bar{Q}_{i}$ are simple Artinian rings. By Theorem [2, Theorem 4.1], $\left|\max \cdot \operatorname{Den}_{l}(\bar{R})\right|=s$. Let $\max \cdot \operatorname{Den}_{l}(\bar{R})=$ $\left\{T_{1}, \ldots, T_{s}\right\}$

(vi) $\left|\max \cdot \operatorname{Den}_{l}(R)\right| \leq s$ : By (v), $\bar{S} \subseteq T_{i}$ for some $i$. Then $S$ is the largest element with respect to inclusion of the set $\left\{S^{\prime} \in \operatorname{Den}_{l}(R) \mid \pi\left(S^{\prime}\right) \subseteq T_{i}\right\}$ (as $0 \in S S_{1}$ for all distinct $S, S_{1} \in \max ^{-D^{2}}(R)$, by Lemma 4.1.(2), where $S S_{1}$ is the multiplicative submonoid of $R$ generated by $S$ and $\left.S_{1}\right)$. Hence, $\left|\max _{\operatorname{Den}}(R)\right| \leq s$.

Let $R$ be a ring such that its largest left quotient ring $Q_{l}(R)$ is a left Noetherian ring. Define the ring homomorphism

$$
p_{i}: R \stackrel{\pi}{\rightarrow} \bar{R} \stackrel{\bar{\sigma}}{\rightarrow} \bar{Q}=\prod_{j=1}^{s} \bar{Q}_{i} \stackrel{\bar{p}_{i}}{\rightarrow} \bar{Q}_{i} .
$$

Proof of Corollary 1.5. 1. The set $D_{i}$ is a nonempty set since $R^{*} \subseteq D_{i}$. By Lemma 4.1.(2), the set $S_{i}^{\prime}:=\bigcup_{S^{\prime} \in D_{i}} S^{\prime}$ is the largest element of the set $D_{i}$. Let $\mathcal{M}$ be the set of maximal elements of the set $\left\{S_{1}^{\prime}, \ldots, S_{s}^{\prime}\right\}$. Then $\max \operatorname{Den}_{l}(R) \subseteq \mathcal{M}$, see the proof of the statement (iv) of Theorem 1.3. The reverse inclusion is obvious.

2. By Theorem 2.3.(2b), $\widetilde{S}_{l} \in \operatorname{Den}_{l}(\bar{R}, 0)$ and $\widetilde{S}_{l} \subseteq \overline{\mathcal{C}}$. By Theorem 2.3.(3), the ring $\bar{R}$ is a semiprime left Goldie ring and $\bar{Q}$ is a semisimple ring. By Theorem 2.1.(1), $\overline{\mathcal{C}}=\bar{R} \cap \bar{Q}^{*}$. Hence, $S_{l} \subseteq S_{i}^{\prime}$ for all $i=1, \ldots, s$. 
3. Statement 3 follows from statement 2: The ring $S_{i}^{\prime-1} R$ is a localization of the left Noetherian ring $S_{l}^{-1} R$ (see statement 2). Hence it is a left Noetherian ring.

Criterion for $\left|\max . \operatorname{Den}_{l}(\boldsymbol{R})\right|=\left|\max \cdot \operatorname{Den}_{l}(\overline{\boldsymbol{R}})\right|$ Let $R$ be a ring such that its largest left quotient ring $Q_{l}(R)$ is a left Noetherian ring. In general, $\left|\max _{\operatorname{lon}}(R)\right| \leq\left|\max _{l} \cdot \operatorname{Den}_{l}(\bar{R})\right|$, Theorem 1.3. The next theorem is a criterion for $\left|\max _{\operatorname{Den}}(R)\right|=\left|\max \cdot \operatorname{Den}_{l}(\bar{R})\right|$.

Theorem 4.2 Let $R$ be a ring such that its largest left quotient ring $Q_{l}(R)$ is a left Noetherian ring. Then $\left|\max _{\text {Den }}(R)\right|=\mid$ max.Den $_{l}(\bar{R}) \mid$ iff for each pair of indices $i \neq j$ where $1 \leq i, j \leq s=\left|\max _{\operatorname{Den}}(\bar{R})\right|$ there exist $S_{i}, S_{j} \in \operatorname{Den}_{l}(R)$ such that $0 \in S_{i} S_{j}$ (where $S_{i} S_{j}$ is the multiplicative submonoid of $R$ generated by $S_{i}$ and $\left.S_{j}\right), p_{i}\left(S_{i}\right) \subseteq \bar{Q}_{i}^{*}$ and $p_{j}\left(S_{j}\right) \subseteq \bar{Q}_{j}^{*}$. In this case, max.Den $(R)=\left\{S_{1}^{\prime}, \ldots, S_{s}^{\prime}\right\}$ where $S_{i}^{\prime}$ is the largest element in the set $\left\{S^{\prime} \in \operatorname{Den}_{l}(R) \mid p_{i}\left(S^{\prime}\right) \subseteq \bar{Q}_{i}^{*}\right\}$.

Proof By [2, Theorem 4.1], $\max _{\operatorname{Den}}(\bar{R})=\left\{T_{1}, \ldots, T_{s}\right\}$ where $T_{i}=\left(\bar{p}_{i} \sigma\right)^{-1}\left(\bar{Q}_{i}^{*}\right)$ for $i=1, \ldots, s$.

$(\Rightarrow)$ It suffices to take $S_{i}=S_{i}^{\prime}, i=1, \ldots, s$ since $0 \in S_{i}^{\prime} S_{j}^{\prime}$ for all $i \neq j$, by Corollary 1.5 and Lemma 4.1.(2).

$(\Leftarrow)$ Suppose that $S_{1}, \ldots, S_{s}$ are as in the theorem. For each $i=1, \ldots, s$, let $S_{i}^{\prime}$ be the largest element with respect to inclusion of the set $\left\{S^{\prime} \in \operatorname{Den}_{l}(R) \mid \pi\left(S^{\prime}\right) \subseteq T_{i}\right\}$. Since $S_{i} \subseteq S_{i}^{\prime}$ and $S_{j} \subseteq S_{j}^{\prime}$ for each distinct pair $i \neq j$ and $0 \in S_{i} S_{j}$, the elements $S_{1}^{\prime}, \ldots, S_{s}^{\prime}$ are distinct (Lemma 4.1.(2)) and incomparable (i.e. $S_{i}^{\prime} \nsubseteq S_{j}^{\prime}$ for all $i \neq j$ ). In the proof of Theorem 1.3, the statement (vi), we have seen that $\max _{\operatorname{Den}}(R) \subseteq\left\{S_{1}^{\prime}, \ldots, S_{s}^{\prime}\right\}$. Hence, $\max _{\operatorname{Den}}(R)=\left\{S_{1}^{\prime}, \ldots, S_{s}^{\prime}\right\}$ since the sets $S_{1}^{\prime}, \ldots, S_{s}^{\prime}$ are incomparable.

Atomic, Orthogonal Subsets of $\operatorname{Den}_{l}(R)$ Let $R$ be a ring and $S \in \operatorname{Den}_{l}(R)$. Let

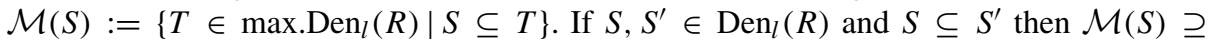
$\mathcal{M}\left(S^{\prime}\right)$. If $S_{i} \in \operatorname{Den}_{l}(R)$ where $i=1, \ldots, n$ then $\mathcal{M}\left(S_{1} \cdots S_{n}\right)=\cap_{i=1}^{n} \mathcal{M}\left(S_{i}\right)$. If $S_{i} \in \operatorname{Den}_{l}(R)$ where $i \in I$ then $\mathcal{M}\left(\vee_{i \in I} S_{i}\right)=\cap_{i \in I} \mathcal{M}\left(S_{i}\right)$.

Lemma 4.3 Let $R$ be a ring and $S, T \in \operatorname{Den}_{l}(R)$. The following statements are equivalent.

1. $0 \in S T$.

2. $\mathcal{M}(S) \cap \mathcal{M}(T)=\emptyset$.

3. There is not a left denominator set of $R$ that contains both $S$ and $T$.

Proof $\left(1 \Rightarrow 3\right.$ ) Suppose that $A \in \operatorname{Den}_{l}(R)$ such that $S, T \subseteq A$. Then $0 \in S T \subseteq A$, a contradiction.

$(3 \Rightarrow 2)$ This implication is obvious.

$(2 \Rightarrow 1)$ If $0 \notin S T$ then $S T \in \operatorname{Den}_{l}(R)$. Let $A \in \mathcal{M}(S T)$. Then $S, T \subseteq S T \subseteq A$, and so $A \in \mathcal{M}(S) \cap \mathcal{M}(T)$, a contradiction.

Definition. Elements $S, T \in \operatorname{Den}_{l}(R)$ that satisfy Lemma 4.3 are called orthogonal. Left denominaor sets $\left\{S_{i}\right\}_{i \in I}$ of $R$ are called orthogonal if $S_{i}$ and $S_{j}$ are orthogonal for all distinct $i$ and $j$.

Example. For an arbitrary ring $R$, the set $\max \operatorname{Den}_{l}(R)$ is orthogonal.

Example. Let $R=R_{1} \times \cdots \times R_{n}$ be a direct product of rings $R_{i}$ and $1=e_{1}+\cdots+e_{n}$ be the corresponding sum of central orthogonal idempotents. Then $S_{i}:=\left\{1, e_{i}\right\} \in \operatorname{Den}_{l}(R)$ for $i=1, \ldots, n$ and $\left\{S_{1}, \ldots, S_{n}\right\}$ is an orthogonal set. 
Definition. A subset $\left\{S_{i}\right\}_{i \in I}$ of $\operatorname{Den}_{l}(R)$ is called a basis of $\operatorname{Den}_{l}(R)$ if $\max \cdot \operatorname{Den}_{l}(R)=$ $\bigsqcup_{i \in I} \mathcal{M}\left(S_{i}\right)$ (a disjoint union) and $\left|\mathcal{M}\left(S_{i}\right)\right|=1$ for all $i \in I$.

Example. For an arbitrary ring $R$, the set $\max \operatorname{Den}_{l}(R)$ is a basis for $\operatorname{Den}_{l}(R)$.

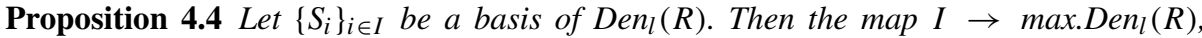
$i \mapsto \mathcal{M}\left(S_{i}\right)$, is a bijection. In particular, $\operatorname{card}(I)=\operatorname{card}\left(\max \operatorname{Den}_{l}(R)\right)$.

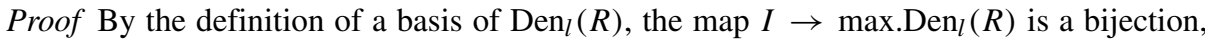
and so $\operatorname{card}(I)=\operatorname{card}\left(\max \cdot \operatorname{Den}_{l}(R)\right)$.

Definition. The cardinality of any basis of $\operatorname{Den}_{l}(R)$ is called the left localization number of $R$ denoted by $\ln (R)$. So, $\ln (R)=\operatorname{card}\left(\max _{\operatorname{Den}}(R)\right)$.

Example. Let $R$ be a commutative ring. Then $\max \cdot \operatorname{Den}_{l}(R)=\left\{S_{\mathfrak{p}}:=R \backslash \mathfrak{p} \mid \mathfrak{p} \in \operatorname{Min}(R)\right\}$ and so $\ln (R)=\operatorname{card}(\operatorname{Min}(R))$.

Example. Let $R$ be a semiprime left Goldie ring and $Q(R)=\prod_{i=1}^{s} Q_{i}$ is a direct product of simple Artinian rings $Q_{i}$. Then $\ln (R)=s=\operatorname{card}(\operatorname{Min}(R))$, [2].

Definition. A left denominator set $S \in \operatorname{Den}_{l}(R)$ is called atomic if $|\mathcal{M}(S)|=1$. A subset of left denominator sets is called atomic if all its elements are atomic.

If $\mathcal{S}$ is an atomic and orthogonal subset of $\operatorname{Den}_{l}(R)$ then the map $v_{\mathcal{S}}: \mathcal{S} \rightarrow$ $\max \operatorname{Den}_{l}(R), S \mapsto \mathcal{M}(S)$, is an injection.

Lemma 4.5 Every atomic, orthogonal subset $\mathcal{S}$ of $\operatorname{Den}_{l}(R)$ can be extended to a basis of $\operatorname{Den}_{l}(R)$. Furthermore, $\mathcal{S} \amalg\left(\right.$ max.Den $_{l}(R) \backslash \operatorname{im}\left(v_{\mathcal{S}}\right)$ is a basis of $\operatorname{Den}_{l}(R)$, In particular, $\operatorname{card}(\mathcal{S}) \leq \ln (R)$.

Proof Straightforward.

Open Access This article is distributed under the terms of the Creative Commons Attribution 4.0 International License (http://creativecommons.org/licenses/by/4.0/), which permits unrestricted use, distribution, and reproduction in any medium, provided you give appropriate credit to the original author(s) and the source, provide a link to the Creative Commons license, and indicate if changes were made.

\section{References}

1. Bavula, V.V.: The algebra of integro-differential operators on an affine line and its modules. J. Pure Appl. Algebra 217, 495-529 (2013). arXiv:math.RA.1011.2997

2. Bavula, V.V.: New criteria for a ring to have a semisimple left quotient ring. J. Alg. Appl. 6(6), 28 (2015). doi:10.1142/S0219498815500905. arXiv:math.RA:1303.0859

3. Bavula, V.V.: The largest strong left quotient ring of a ring. J. Algebra 439, 1-32 (2015). arXiv:math.RA.1310.1077

4. Bavula, V.V.: The largest left quotient ring of a ring. Commun. Algebra 44(18), 3219-3261 (2016). arXiv:math.RA.1101.5107

5. Bavula, V.V.: Left localizations of left Artinian rings. J. Alg. Appl. 15(9), 1650165, 38 (2016). doi:10.1142/S0219498816501656. arXiv:math.RA:1405.0214

6. Bavula, V.V.: Criteria for a ring to have a left Noetherian left quotient ring. J. Pure Appl. Alg., to appear arXiv:math.RA.1508.03798

7. Birkenmeier, G.F., Park, J.K., Rizvi, S.T.: Ring hulls and applications. J. Algebra 304, 633-665 (2006)

8. Chatters, A.W., Hajarnavis, C.R.: Quotient rings of Noetherian module finite rings. Proc. AMS 121(2), 335-341 (1994)

9. Jategaonkar, A.V.: Localization in noetherian rings. Londom Mathematics Society. LMS 98, Cambridge University (1986) 
10. McConnell, J.C., Robson, J.C.: Noncommutative Noetherian rings. With the cooperation of L. W. Small. Revised edition. Graduate Studies in Mathematics, 30, p. 636. American Mathematical Society, Providence, RI (2001)

11. Michler, G., Müller, B.: The maximal regular Ore set of a Noetherian ring. Arch. Math. 43, 218-223 (1984)

12. Stafford, J.T.: Noetherian full quotient rings. Proc. Lond. Math. Soc. (3) 44, 385-404 (1982)

13. Stenström, B.: Rings of quotients. Springer-Verlag, Berlin, Heidelberg New York (1975) 